\title{
Aprovechamiento tradicional de mamíferos silvestres en Pitzotlan, Morelos, México
}

\section{Traditional use of wild mammals in Pitzotlan, Morelos, Mexico}

\author{
García F, Alejandro' Ph.D; Valle M, Raúl ${ }^{2}$ Biol; Monroy M, Rafael ${ }^{1}$ M.Sc.
}

1 Universidad Autónoma del Estado de Morelos. Centro de Investigaciones Biológicas. Laboratorio de Ecología. Morelos, México. 2Universidad Autónoma del Estado de Morelos. Facultad de Ciencias Biológicas. México. Av. Universidad 1001. Colonia Chamilpa, Código Postal 62209.

\section{KEYWORDS:}

Traditional knowledge; ethnozoology; managements; use values.

\section{PALABRAS CLAVE:}

Conocimiento tradicional; etnozoología;

manejo;

valores de uso.
INFORMACIÓN

Recibido: 28-12-2017; Aceptado: 02-05-2018.

Publicado: 02-07-2018

Correspondencia autor: alejandro.garcia@uaem.mx

\section{ABSTRACT}

The ethnozoological knowledge regarding wild mammals that the inhabitants of Pitzotlán preserve is analyzed. By the application of ethnozoological techniques we registered the use of 6 orders, 11 families and 20 species, $55 \%$ of which had the following use value: as foodstuff $(100 \%)$, medicinal $(45 \%)$, as pets $(18 \%)$, ornamental $(18 \%)$, as goodluck charms (18\%) and in tool manufacturing (9\%). Its exploitation is done by means of subsistence hunting; in this case guns $(100 \%)$, dogs $(36 \%)$ and slings (18\%) are used. The white-tailed deer and the badger are the most used species; they register an index of cultural importance of value 1 . Due to different actions of habitat enhancement as well as the implementation of wildlife management standards, the inhabitants have registered a rise in the populations of white-tailed deer and boar. We discuss the importance of mammals, as an edible and medicinal resource, as well as the importance of recovering and appreciating the traditional knowledge with the participation of the population so as to generate conservation strategies and rational exploitation plans of these mammals for the benefit of the community.

\section{RESUMEN}

Se analiza el conocimiento etnozoológico que conservan los habitantes de Pitzotlán, relativo a los mamíferos silvestres. Mediante técnicas etnozoológicas se registró el aprovechamiento de 6 órdenes, 11 familias y 20 especies, de las cuales el $55 \%$ de las especies tienen los siguientes valores de uso: alimentario $(100 \%)$, medicinal $(45 \%)$, animales de compañía $(18 \%)$, ornamental $(18 \%)$, amuleto $(18 \%)$ y en la fabricación de herramientas (9\%). Su explotación se lleva a cabo mediante la cacería de subsistencia, en este caso se utilizan armas de fuego $(100 \%)$, perros $(36 \%)$ y resorteras $(18 \%)$. El venado cola blanca y el tejón fueron las especies más utilizadas, registrando un Índice de importancia cultural de 1 . Debido a diferentes acciones de mejoramiento del hábitat y a la implementación de normas para el manejo de la fauna, los habitantes han registrado un incremento en las poblaciones de venado cola blanca, tejón y jabalí. Se discute la importancia de los mamíferos como recurso alimentario y medicinal, así como rescatar y valorar el conocimiento tradicional con la participación de la población para generar estrategias de conservación y formas de aprovechamiento racional de estos mamíferos, en beneficio de la comunidad. 


\section{INTRODUCCIÓN}

La fauna silvestre (FS), constituye un componente en la estructura y dinámica de los ecosistemas al cumplir funciones en procesos como el flujo de energía, dispersión de semillas y polinización de plantas, además es uno de los recursos importantes para la subsistencia de diversos grupos sociales (HERNÁNDEZ et al., 2013; LIRA et al., 2014).

El aprovechamiento de la fauna desempeña un papel fundamental en el desarrollo social y económico de la sociedad, coadyuvando a resolver las necesidades alimentarias, medicinales, culturales (RETANA et al., 2011) e ingresos económicos mediante su mercadeo (MONROY y GARCÍA, 2013). Históricamente, los mamíferos silvestres han sido utilizados como alimento, resolviendo la problemática relativa a la ingesta de proteínas de las comunidades campesinas (MONROY et al., 2008; LIRA et al., 2014), aunque también se ha evidenciado que son aprovechados con otros fines como el estético, ornamental y religioso (GARCÍA et al., 2014).

La FS y el conocimiento tradicional ligado a las especies de animales, están sometidos a factores inducidos por el sistema económico, el cual ha provocado su pérdida y deterioro. El modelo de desarrollo capitalista induce la fragmentación de los ecosistemas, por lo que la diversidad, distribución y abundancia de la fauna en América Latina han disminuido (GUERRA et al., 2010; RODAS et al., 2014), por lo que este modelo representa una de las principales amenazas para el patrimonio biocultural de la población campesina e indígena que depende de la fauna para obtener alimento e ingresos económicos que complementan la canasta básica alimentaria (GONZÁLEZ y LLERENA, 2014).

Ante esta situación el objetivo fue analizar el conocimiento etnozoológico que conservan los habitantes sobre los mamíferos silvestres de Pitzotlan, Morelos, México.

\section{MATERIAL Y MÉTODOS}

Área de estudio. El ejido de Pitzotlan, se localiza en el municipio de Tepalcingo en el estado de Morelos, México. Geográficamente, en las coordenadas $18^{\prime} 34^{\prime} 58^{\prime \prime}$ de LN y $98^{\prime} 53^{\prime} 29^{\prime \prime}$ de LO (Figura 1), a una altitud de 1.178 metros sobre el nivel del mar y cuenta con una superficie de 1.009 hectáreas. La comunidad presenta una población mestiza de 39 habitantes de los cuales 26 son hombres y 13 mujeres (INEGI, 2010), clasificados en la categoria de muy alta marginación (MUNDO, 2011).

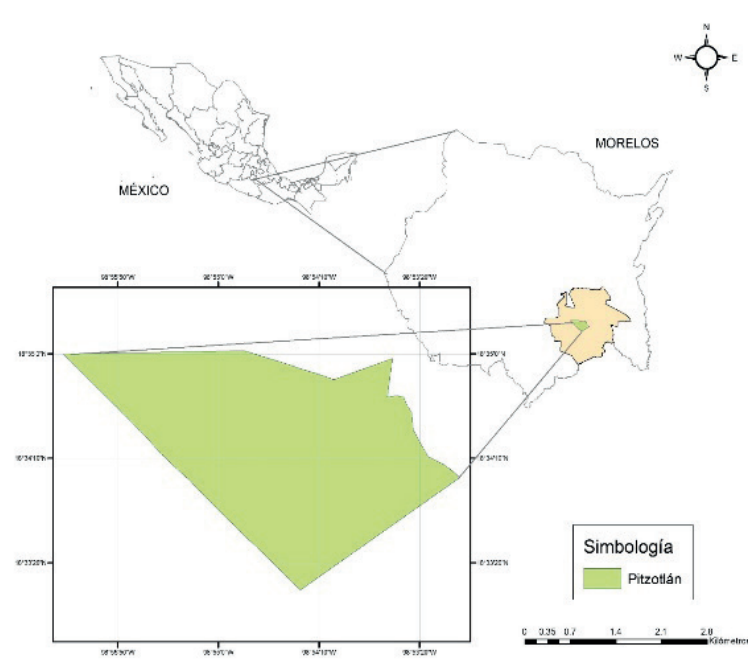

Figura 1. Ubicación de Pitzotlan, Tepalcingo, Morelos, México (INEGI, 2010).

Las actividades económicas del ejido son la ganaderia extensiva de bovinos, equinos, ovinos y la agricultura de temporal. Se cultiva maíz (Zea mays L. 1753), frijol (Phaseolus vulgaris L. 1753) y calabaza (Cucurbita pepo L. 1753), cuya produccion se destina al autoabasto, al igual que la ganadería de traspatio donde se crían gallinas (Gallus gallus), guajolotes (Meleagris gallopavo), cerdos (Sus scrofa) y borregos (Ovis aries). Otra actividad importante es la colecta de pitaya (Stenocereus stellatus) que se encuentra en estado silvestre y cultivada en huertos, la cual se comercializa a nivel local y regional. 
El clima corresponde al tipo Aw0' '(w) (e) $\mathrm{g}$ calido con lluvias en verano, el mas seco de los subhúmedos con canícula, \% de Iluvia invernal menor de cinco, extremoso y marcha de temperatura tipo Ganges. La temperatura media anual es de $22^{\circ} \mathrm{C}$ y precipitación de 951 mm (TABOADA et al., 2009).

La vegetación del área de estudio es la selva baja caducifolia (MIRANDA y HERNÁNDEZ, 1963), caracterizada por comunidades dominadas por árboles de baja altura, donde la mayor parte de la vegetación pierde las hojas en la época seca.

Como primer actividad se contacto a la autoridad local (Ayudante Municipal), tal como lo sugieren TAYLOR y BOGDAN (1986), al que se le informó sobre la investigación y los objetivos a desarrollar lo cual nos permitio obtener el permiso para ingresar a la comunidad, además fue él quien convoco a los pobladores para hacer de su conocimiento el proyecto.

Se utilizó la técnica del guia clave, el cual de acuerdo a ROBLEDO (2009) son aquellas personas que por sus vivencias, capacidad de empatizar y relaciones sociales que tienen en el campo pueden ayudar al investigador conviritiéndose en una fuente importante de conocimiento a la vez que nos facilita el acceso a otras personas y nuevas experiencias. Con esta premisa, se seleccionó a la persona que cumplió dichas carácterísticas, este fué el ayudante de la comunidad, para despúes utilizar la técnica de bola de nieve o muestreo en cadena (MARTÍN y SALAMANCA, 2007). El muestreo se considero terminado cuando ya no se obtuvo información relevante o nueva para la investigación.

Un elemento importante en la investigación fue el establecimiento de rapport, el cual no es un concepto que pueda definirse facilmente como menciona TAYLOR y BOGDAN (1986), pero se define como lograr una relación de confianza que permité a las personas apoyar al investigador (ROBLEDO 2009). De esta manera se creó un ambiente de confianza con los colaboradores claves y posteriormente con las demás personas que participaron especialmente durante las entrevistas.
La sistematización del conocimiento, valor de uso y manejo de los mamiferos se llevó a cabo mediante la técnica de la entrevista, la cual es definida por HAGUETTE (1992) como el proceso de interacción social entre dos personas en la que una de ellas, el entrevistador tiene como objetivo la obtención de información por parte del otro, el entrevistado. La modalidad de entrevista que se utilizó fue la semiestructurada, en las cuales los tópicos fueron previamente conocidos y otros se redifinideron durante el desarrollo de la investigación, con la finalidad de adecuarse a las necesidades del entrevistador en cada momento. Se aplicaron entrevistas al $100 \%$ de hombres $(n=11)$ que practican o han desarrollado la cacería en la comunidad. Las entrevistas fueron grabadas con el consentimiento de los informantes con una grabadora portátil marca TASCAM, modelo DR-05, estas tuvieron una duración de 1:30-2:40 h y se realizaron en las casas de los entrevistados en compañía de la esposa, hijos, nueras o nietos, los cuales aportaron información sobre el aprovechamiento de los mamíferos silvestres.

Otra técnica que se utilizó para la obtención de la información fue la observación participante, la cual se define como el proceso de aprendizaje a través del involucramiento en las actividades productivas y culturales de los participantes en el escenario del investigador (ROBLEDO, 2009).

La información que se registró en las entrevistas fue la referente a las características socioeconómicas de los informantes, nombre, edad, sexo, tiempo de residencia, escolaridad, ocupación, integrantes de la familia; así como información repecto a los mamíferos silvestres conocidos, valores de usos, partes usadas, época de aprovechamiento, tiempo y frecuencia de caza, especies preferidas para cazar, métodos e instrumentos de cacería, lugares de caza, conocimiento sobre la biología de las especies, percepción sobre la preservación de mamíferos, su importancia y propuestas de manejo y conservación.

Las especies de mamíferos que fueron mencionadas por los entrevistados se determinaron con base en la metodología propuesta por MONROY y GARCÍA (2013). Adicionalmente se realizaron recorridos 
guiados para identificar huellas, excretas y marcas territoriales de los mamíferos. También se analizaron trofeos de cacería, extremidades y pieles de animales que poseían los entrevistados en su casa, además, para la identificación de especies se consultaron las guías de campo ARANDA (2000, 2012).

La información obtenida de las entrevistas se sistematizó utilizando el programa Microsoft Excel y se analizó mediante estadistica descriptiva. Para cada especie de mamífero registrada, se cálculo el Índice de Importancia Cultural (IIC) propuesto por PARRA et al. (2014):
$\mathrm{IIC}=(\mathrm{Iu}+\mathrm{Nr}) / \mathrm{Neu} ;$ dónde: $\mathrm{Iu}=$ Intensidad de uso (número de uso); $\mathrm{Nr}=$ Número de reportes; Neu = Número de especies útiles. Este índice va de 0 a 1; siendo 0 cuando no hay uso de la especie y 1 cuando la especie es altamente utilizada en la comunidad.

\section{RESULTADOS}

Los entrevistados reconocieron 20 especies de mamíferos silvestres, agrupadas en 6 órdenes y 14 familias, destacando el orden Carnívora y las familias Felidae y Procyonidae (Tabla 1).

Tabla 1. Mamiferos silvestres de Pitzotlan, Tepalcingo, Morelos.

\begin{tabular}{|c|c|c|c|c|c|c|}
\hline Orden & Familia & Nombre científico & Nombre local & Valor de Uso & Parte usada & *IIC \\
\hline \multirow[t]{4}{*}{ ARTIODACTYLA } & Tayassuidae & Pecarí tajacu & Marrano de monte & Alimento & Carne & 0.27 \\
\hline & & & & Alimento & Carne, vísceras & \\
\hline & Cervidae & Odocoileus virginianus & Venado & & Sangre, grasa & 1 \\
\hline & & & & $\begin{array}{l}\text { Ornamental } \\
\text { Herramienta }\end{array}$ & $\begin{array}{l}\text { Cabeza pıel } \\
\text { Extremidades }\end{array}$ & \\
\hline \multirow[t]{2}{*}{ LAGOMORPHA } & Leporidae & Sylvilagus cunicularius & Conejo & $\begin{array}{l}\text { Alimento } \\
\text { Mascota }\end{array}$ & $\begin{array}{c}\text { Carne } \\
\text { Animal vivo }\end{array}$ & 0.63 \\
\hline & & Lepus callotis & Liebre & Alimento & Carne & 0.09 \\
\hline \multirow[t]{19}{*}{ CARNIVORA } & Felidae & Puma concolor & Puma & & & \\
\hline & & Leopardus wiedii & Tigrillo & & & \\
\hline & & Leopardus pardalis & Ocelote & & & \\
\hline & & Puma yagouaroundi & Yaguarundi & & & \\
\hline & & Lynx Rufus & Gato montes & & & \\
\hline & & & & Alimento & Carne & \\
\hline & Canidae & Canis latrans & Coyote & $\begin{array}{c}\text { Medicinal } \\
\text { Ornamental }\end{array}$ & $\begin{array}{c}\text { Carne } \\
\text { Colmillos }\end{array}$ & 0.54 \\
\hline & & & & Amuleto & Colmillos & \\
\hline & & Urocyon cinereoargenteus & Zorra gris & & & \\
\hline & Mustelidae & Mustela frenata & Comadreja & & & \\
\hline & & & & Alimento & Carne & \\
\hline & Procyonidae & Nasua narica & Tejón & Medicinal & Grasa & 1 \\
\hline & & & & Mascota & Animal vivo & \\
\hline & & Procyon lotor & Kailama & Alimento & Carne & 0.09 \\
\hline & & Bassariscus astutus & Cacomixtle & & & \\
\hline & & & & Alimento & Carne & \\
\hline & Mephitıdae & Mephitis macroura & Zorrillo & Medicinal & Carne & 0.63 \\
\hline & & Conepatus leuconotus & Zorrillo cadeno & Alimento & Carne & 0.63 \\
\hline & & & 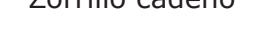 & Medicinal & Carne & \\
\hline \multirow{2}{*}{ DIDELPHIMORPHIA } & Didelphidae & Didelphis virainiana & Tlacuache & Alimento & Carne & 027 \\
\hline & Dideriptide & Diaeipmis Virginama & macuacne & Amuleto & Extremidades & 0.27 \\
\hline CINGULATA & Dasypodidae & Dasypus novemcinctus & Armadillo & Alimento & Carne & 0.09 \\
\hline RODENTIA & Sciuridae & Sciurus aureogaster & Ardilla & & & \\
\hline
\end{tabular}


El aprovechamiento de los mamíferos silvestres en este ejido se da por medio de la cacería de subsistencia, la cual se practica por los pobladores de la comunidad para satisfacer sus necesidades y la de sus familias, como son las alimentarias y medicinales.

Se registraron tres tipos de armas utilizadas en la cacería, siendo el arma de fuego (escopeta) la más utilizada para la captura de presas (Figura 2). De acuerdo con los informantes en la comunidad se practican cuatro técnicas para llevar a cabo el aprovechamiento de los mamíferos silvestres, las más utilizadas es la espiada y arreada, las cuales fueron referidas por el $100 \%$ de los cazadores (Figura 3).

Son tres lugares donde se lleva a cabo el aprovechamiento de los mamíferos silvestres, monte y los cultivos (100\%) como las zonas preferidas para llevar cabo la actividad de la caza y los traspatios (70\%).

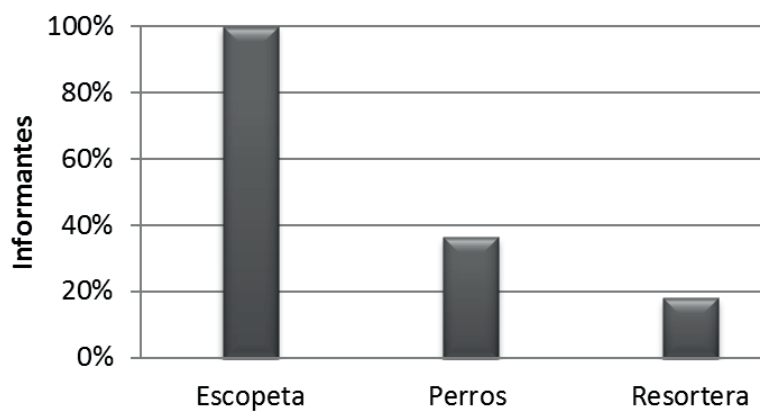

Figura 2. Armas utilizadas para la captura de mamíferos silvestres

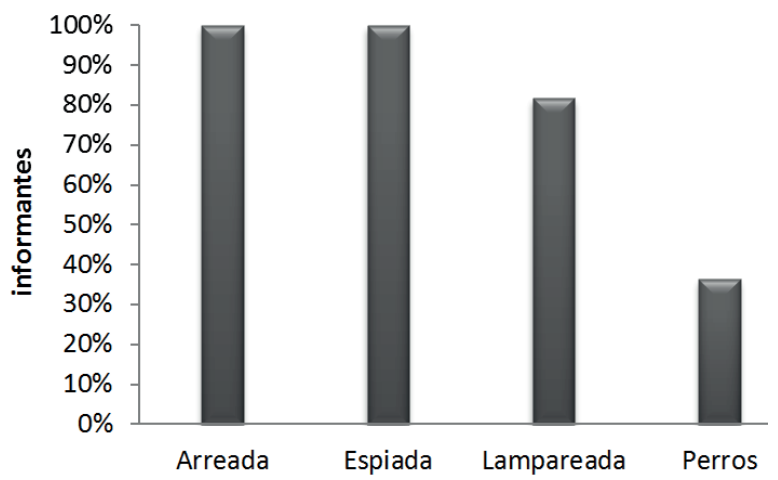

Figura 3. Técnicas de cacería utilizadas por los entrevistados.
Del total de especies reconocidas para el ejido por los entrevistados $(n=20$ especies), el $55 \%(n=11)$ tienen valor de uso (Tabla 1), siendo más representativo el orden Carnívora y las familias Procyonidae, Mephitidae, Leporidae por el número de especies registradas con valor de uso (VU). Las especies con mayor valor de uso fueron el venado cola blanca Odocoileus virginianus (IIC=1) y tejón Nasua narica (IIC=1).

Se registraron seis valores de uso para los mamíferos silvestres: alimento, medicina, animal de compañía, ornamental, amuleto y fabricación de herramientas, destacando el alimentario y medicinal con el mayor número de especies, con 11 y 5 respectivamente (Figura 4).

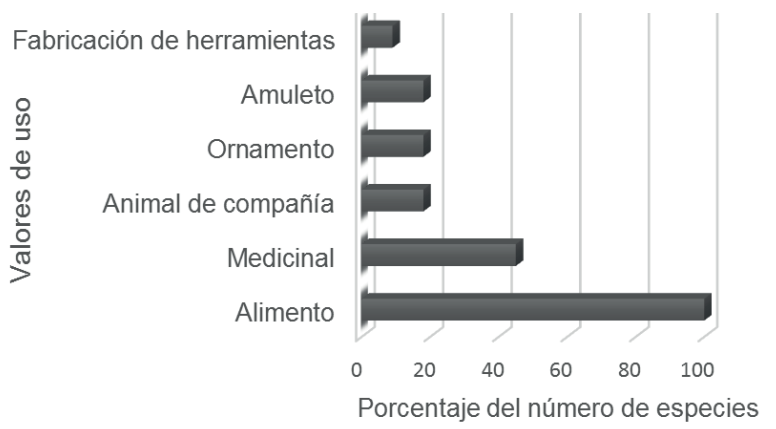

Figura 4. Valores de uso de los mamíferos silvestres en Pitzotlan.

El uso alimentario se basa en el consumo de carne y vísceras de los animales silvestres, los cuales se preparan en 10 platillos tradicionales de la comunidad (Tabla 2).

Para el valor de uso medicinal se utiliza la sangre, carne y grasa para el tratamiento de enfermedades en las personas, las cuales fueron clasificadas para esta investigación con base en la Organización Panamericana de la Salud (2003) en: 33\% por padecimientos ligados al sistema respiratorio, seguidas de las enfermedades del sistema circulatorio, nervioso, urinario, piel y tejido subcutáneo con un $16.7 \%$ cada una. Se reportan cinco especies para curar afecciones como la bronquitis, el asma, granos, manchas en la piel, alergias, presión alta, epilepsia, reuma, dolores musculares, tisis y los problemas renales (Tabla 3). 
Tabla 2. Platillos tradicionales preparados con mamíferos silvestres.

\begin{tabular}{|c|c|c|}
\hline Platillo & $\begin{array}{l}\text { Parte } \\
\text { usada }\end{array}$ & $\begin{array}{c}\text { Mamíferos } \\
\text { utilizados }\end{array}$ \\
\hline Guaxmole & Carne & Venado \\
\hline Barbacoa o tlaquemal & Carne & $\begin{array}{c}\text { Jabalí, venado, } \\
\text { tejón, conejo, } \\
\text { tlacuache, } \\
\text { mapache }\end{array}$ \\
\hline Caldo rojo o verde & Carne & Venado \\
\hline Bistecs y Cecina & Carne & Venado \\
\hline Salsa verde o roja & Carne & Venado y tejón \\
\hline Mole & Carne & $\begin{array}{c}\text { Venado, tejón y } \\
\text { zorrillo }\end{array}$ \\
\hline Adobo & Carne & Venado y tejón \\
\hline Carne frita & Carne & Venado y tejón \\
\hline Carne seca & Carne & Venado \\
\hline Asadura & Vísceras & Venado \\
\hline
\end{tabular}

Los animales de compañía son aquellas especies que los informantes capturan para mantener dentro de jaulas en sus hogares o libres en el traspatio, incluso son animales que consideran "de lujo". Se reportaron dos especies con este uso: conejo Sylvilagus cunicularius que son capturados para mantenerlos en corrales en las casas y el tejón Nasua narica, el cual se captura en la etapa de cría para acostumbrarlo al hogar, sin embargo, mencionan que es un animal sensible a personas ajenas del hogar, ya que es muy susceptible a recibir "mal de ojo".

Se utilizan las estructuras de los mamíferos silvestres, tales como cráneos y pieles, para elaborar objetos o prendas destinadas a la decoración del hogar o de las personas. Se registraron dos especies con este valor: El venado $O$. virginianus su piel se utiliza para confeccionar prendas de vestir como chalecos o se regalan a amigos o familiares fuera del pueblo. Uno de los usos más extendidos de esta especie en la comunidad es el uso de su cabeza como trofeo y perchero. En el caso del coyote Canis latrans se utilizan los colmillos para adornar collares.

Se registraron dos especies que aportan estructuras utilizadas como amuleto para atraer buena suerte o evitar los daños. Los colmillos del coyote Canis latrans, se utiliza para proteger a los niños contra el "mal de ojo" y al tlacuache Didelphis virginiana se le amarra un moño rojo en la pata o mano del animal, se le coloca una moneda y se

Tabla 3. Mamíferos silvestres medicinales.

\begin{tabular}{|c|c|c|c|c|c|}
\hline $\begin{array}{l}\text { Nombre } \\
\text { Común }\end{array}$ & Enfermedad & OPS & $\begin{array}{l}\text { Parte } \\
\text { usada }\end{array}$ & $\begin{array}{c}\text { Tipo de } \\
\text { aplicación }\end{array}$ & Forma de preparación \\
\hline & $\begin{array}{l}\text { Enfermedades } \\
\text { del corazón e } \\
\text { hipertensión }\end{array}$ & $\begin{array}{l}\text { Sistema } \\
\text { Circulatorio }\end{array}$ & Sangre & Ingerida & $\begin{array}{c}\text { Recién cazado el animal, se bebe } \\
\text { su sangre o se deja coagular y } \\
\text { posteriormente se licua junto a jugo } \\
\text { de frutas y se toma. }\end{array}$ \\
\hline \multirow[t]{2}{*}{$\begin{array}{l}\text { Venado cola } \\
\text { blanca }\end{array}$} & Bronquitis y asma & $\begin{array}{c}\text { Sistema } \\
\text { Respiratorio }\end{array}$ & Grasa & Tópico & $\begin{array}{c}\text { Se rasca el cebo de la piel del venado } \\
\text { y este se usa como ungüento sobre el } \\
\text { pecho de la persona enferma. }\end{array}$ \\
\hline & Epilepsia & $\begin{array}{l}\text { Sistema } \\
\text { Nervioso }\end{array}$ & Sangre & Ingerida & $\begin{array}{c}\text { Se toma la sangre del animal recién } \\
\text { cazado. }\end{array}$ \\
\hline Zorrillos & $\begin{array}{l}\text { Granos en la piel y } \\
\text { alergias en general }\end{array}$ & $\begin{array}{l}\text { Piel y tejido } \\
\text { subcutáneo }\end{array}$ & Carne & Ingerida & $\begin{array}{l}\text { Para tratar estos padecimientos se } \\
\text { consume la carne frita del zorrillo. }\end{array}$ \\
\hline Tejón & $\begin{array}{c}\text { inflamación en los } \\
\text { riñones }\end{array}$ & $\begin{array}{l}\text { Sistema } \\
\text { urinario }\end{array}$ & Grasa & Tópico & $\begin{array}{c}\text { El animal se abre recién cazado y la } \\
\text { parte de los riñones se asienta sobre } \\
\text { la grasa del animal o también se } \\
\text { elaboran plastos de hojas de higuerilla } \\
\text { junto con la grasa y se colocan sobre } \\
\text { la parte afectada }\end{array}$ \\
\hline Coyote & Tisis & $\begin{array}{c}\text { Sistema } \\
\text { Respiratorio }\end{array}$ & Carne & Ingerida & $\begin{array}{l}\text { Para tratar este padecimiento se } \\
\text { consume la carne frita del animal. }\end{array}$ \\
\hline
\end{tabular}

OPS: Organización Panamericana de la Salud (2003) 
guarda en la cartera para atraer la buena fortuna.

Las estructuras animales como piel y extremidades, se utilizan para elaborar utensilios y herramientas que son usadas en las actividades diarias de los cazadores. Solo se registró al venado cola blanca, del cual se usan sus patas para elaborar fuetes para caballos y algunas veces las pieles en la elaboración de correas.

El $100 \%$ de los cazadores señaló que las poblaciones de venado Odocoileus virginianus, tejón Nasua narica y jabalí Pecari tajacu se han incrementado. Entre los factores que se mencionan han influido están: 1. Mayor presencia de cultivos de sorgo, el cual se siembra y cosecha entre los meses de octubre y noviembre, lo que representa un recurso alimenticio para la fauna silvestre, 2. A la organización comunitaria para cuidar que se no practique la caza ilegal por personas ajenas a la comunidad y 3 . El mejoramiento del hábitat de los animales, como la construcción de bebederos y la plantación de pasto para la alimentación del venado.

Los cazadores expresan que para ellos la fauna silvestre es importante, al igual que el hábitat, porque les proporcionan los siguientes servicios ecosistémicos: 1. Provisión: porque les aporta alimento, carne de campo, la cual prefieren sobre carnes procesadas, y medicinas que emplean por la falta de servicios de salud, 2. Regulación: la fauna limpia los terrenos y 3. Culturales: consideran que los mamíferos cumplen una función importante en la estética del paisaje, ya que analizan que se ven bonitos los animales del monte, además de que los consideran como un elemento a presumir - lucir cuando tienen visitas. También mencionan que les proporcionan un motivo para convivir.

Las reglas comunitarias que se han establecido en reuniones entre los cazadores de la comunidad para la regulación del aprovechamiento del venado cola blanca son: 1. Vigilancia permanente para evitar que personas ajenas a Pitzotlan se encuentren en el ejido cazando cualquier animal. Ésta supervisa cuando los habitantes realizan sus actividades cotidianas como pastoreo o agricultura durante la semana y los fines de semana, se asignan turnos para formar cuadrillas de vigilancia y realizar rondines por el ejido. En el caso de encontrar a personas ajenas practicando la caza, los entrevistados mencionan que primeramente se le llama la atención y se les invita a abandonar el ejido o de lo contrario se les llamará a las autoridades, 2 . Un habitante de la comunidad que caza un venado fuera de temporada, se hará acreedor a una multa mínima de $\$ 500.00$ y 3 . Está prohibido cazar hembras o crías de venado, por lo tanto, solo se caza a venados machos.

\section{DISCUSIÓN}

El aprovechamiento de mamíferos silvestres en la comunidad de Pitzotlan es por medio de la cacería de subsistencia, la cual de acuerdo con OJASTI y DALLMEIER (2000) representa para la población mestiza la principal forma de apropiación de la fauna silvestre en América Latina. Para los entrevistados, la apropiación de los mamíferos silvestres tiene como principal objetivo satisfacer sus necesidades y la de sus familias, principalmente la alimentaria, negando que exista comercio con algún producto o subproducto proveniente de las especies de mamíferos, situación que concuerda con el concepto de cacería de subsistencia propuesto por GUERRA y NARANJO (2003), la cual definen como "la extracción de fauna silvestre con fines de autoabasto para solventar las necesidades básicas del cazador y la de sus familias, sin motivos comerciales".

La cacería de subsistencia en el área de estudio es una actividad complementaria, ya que ninguno de los informantes se dedica exclusivamente a ella, sino que desarrollan otras actividades como agricultura, ganadería, pesca y recolección de pitaya y leña, escenario que de acuerdo a TOLEDO et al. (2008) representa la estrategia campesina de uso múltiple de los recursos naturales, tanto para fines de subsistencia como para su intercambio económico local y regional. 
Con base en los datos socioeconómicos obtenidos en la investigación, jóvenes y adultos practican la cacería, ya que una de las característica de la cacería campesina es el arraigo entre jóvenes y mayores de edad como lo menciona MONTIEL et al. (1999), pero también expresan representa un motivo de convivencia y socialización entre los hombres, jóvenes, mujeres y niños de la comunidad, característica que se ha documentado en comunidades rurales en el norte de Yucatán, México (MONTIEL et al., 1999) y en comunidades campesinas en diferentes regiones de Colombia (RACERO y GONZÁLEZ, 2014).

La presente investigación reporta un mayor número de mamíferos aprovechados $(n=11$ especies), en comparación con el estudio realizado en comunidades de la Reserva de la Biosfera Sierra de Huautla, donde se reportan ocho mamíferos silvestres con valor uso (VELARDE y CRUZ, 2015). Sin embargo, en México (GARCÍA et al., 2014), Perú (GONZÁLEZ y LLERENA, 2014) y Colombia (PARRA et al., 2014) se registra una mayor riqueza de especies utilizadas.

En cuanto a los valores de uso se reportan seis para la mastofauna, destacando el alimentario y medicinal con mayor número de especies. A pesar de que las principales necesidades que busca satisfacer la apropiación de fauna silvestre en México por parte de campesinos son la alimentaria y la medicinal (BARRASA, 2012), los cazadores de áreas rurales utilizan a los animales silvestres para obtener productos ornamentales 0 rituales (amuletos), además de tenerlos como mascota e incluso conseguir algunas herramientas (NARANJO et al., 2004), como se reporta en la presente investigación.

Para las comunidades de la Reserva Estatal Sierra de Nanchititla, en el estado de México (MONROY et al., 2008) y en Córdoba, Colombia (RACERO y GONZÁLEZ, 2014) registran valores de uso similares para los mamíferos reportados en este trabajo de investigación, solo difiriendo en la asignación de valor de cambio para ciertas especies de mamíferos.
El uso alimentario representa el valor más importante de los mamíferos para los habitantes de Pitzotlan, siendo esta la forma de aprovechamiento de la fauna silvestre en Latinoamérica, representando una alternativa para el complemento de la alimentación de la población rural por el aporte de proteína animal (GUERRA et al., 2010), aspecto importante al considerar en regiones rurales de México, debido a que el consumo de proteína animal proveniente de fauna silvestre aporta hasta el $70 \%$ de la ingesta proteínica humana (INE, 1997), mientras que en poblaciones rurales sudamericanas se ha estimado que oscila entre un $30-50 \%$ de la proteína animal consumida por sus habitantes proviene de animales silvestres (STEARMAN y REDFORD, 1995). El consumo de armadillo, jabalí, liebre, mapache, tejón y tlacuache, también es reportado para otras comunidades de México (RODAS et al., 2014).

La especie con mayor frecuencia de valor de uso alimentario fue el venado cola blanca (Odocoileus virginianus), también reportado por ESCAMILLA et al. (2000), quienes lo mencionan como fuente de proteína de origen animal. Uno de los criterios que los informantes citan para preferir el consumo de carne de venado es su sabor, ya que la consideran muy sabrosa, el consumo de este animal está muy arraigado en diferentes comunidades de México (LÓPEZ et al., 2005).

El uso medicinal de los mamíferos, representa el segundo valor de uso más importante en la comunidad, éste es un aspecto relevante a considerar debido a que dentro de la comunidad no existen servicios de salud, RODAS et al. (2014) han mencionado que el uso de animales con fines medicinales es una práctica cotidiana en diversas comunidades, ya sea por no tener acceso a la medicina convencional o por tradición. En el caso del venado cola blanca, el tratamiento de enfermedades respiratorias como bronquitis y asma por medio de su grasa es similar a lo reportado por RETANA et al. (2015) en comunidades del norte de Campeche; y en los altos de Chiapas el venado es usado para el tratamiento de enfermedades del corazón 
(ENRÍQUEZ et al., 2006), sin embargo, se utilizan las astas en polvo en lugar de beber la sangre como se reporta en el presente estudio.

El uso de las especies de zorrillos para el tratamiento de afecciones en la piel es frecuentemente documentado en diferentes sitios de México (GARCÍA et al., 2014; VELARDE y CRUZ, 2015; AMADOR y DE LA RIVA, 2016).

Es importante mencionar que los animales no se capturan de manera exclusiva para obtener estructuras para ornamentos y elaboración de herramientas. En cuanto a las especies utilizadas para fabricar herramientas, RETANA et al., (2015), reportan que, en comunidades del norte de Campeche, México se utilizan las extremidades inferiores para la elaboración de fuetes, práctica que coincide en la comunidad de Pitzotlan. Uno de los usos ornamentales más extendido en la comunidad de estudio, es el uso de la cabeza, piel y astas del venado cola blanca, principalmente como adorno en los hogares, lo cual representa una práctica común en diversas localidades de México (AMADOR y DE LA RIVA, 2016).

En lo que respecta a mamíferos usados como animales de compañía, estos también han sido reportados como mascota en comunidades de otros estados de la República Mexicana (Barrasa, 2012; Lira et al., 2014).

La escopeta fue el principal instrumento de apropiación de la mastofauna local, arma que caracteriza a la cacería de subsistencia, lo cual ha sido reportado en otras regiones de México, Argentina, Colombia y Perú (PAUTASSO, 2003; GONZÁLEZ y LLERENA, 2014; RACERO y GONZÁLEZ, 2014; AMADOR y DE LA RIVA, 2016). Además, se utilizan otros instrumentos como perros y resorteras en combinación con las armas de fuego, por ejemplo, la escopeta junto con perros para la captura de presas grandes y escopeta con resortera para presas chicas, situación que describe AMADOR y DE LA RIVA (2016) en comunidades de Aguascalientes, México. Igualmente, la utilización de perros en conjunto con armas de fuego se ha documentado en distintas zonas de México y Argentina, principalmente porque eleva la eficacia para la apropiación de fauna, al convertir a los perros en rastreadores de las presas y capturándolas con las armas de fuego (PAUTASSO, 2003; HERNÁNDEZ et al., 2013; GARCÍA et al., 2014; LIRA et al., 2014).

Las técnicas de apropiación más utilizadas fueron la arriada y espiada aplicadas en el aprovechamiento del venado, han sido documentadas en el sureste mexicano, las cuales para los cazadores disminuyen el esfuerzo para obtener las presas, (LIRA et al., 2014) y representa un ejemplo de organización social (MONTIEL et al., 1999), ya que como se observó en el área de estudio, existe organización de la actividad de la cacería, desde reuniones previas y punto de reunión, división y organización del trabajo de lo que dependerá el éxito, hasta la asignación de la cacería por parte de los entrevistados como un espacio de esparcimiento, socialización y convivencia entre los integrantes de la comunidad; además de que permite la transmisión del conocimiento de los adultos con experiencia hacia los jóvenes, ya sea en procesos verticales, de padres a hijos, o en horizontales, transmisión entre conocidos.

Las tres áreas de apropiación de la mastofauna registradas (monte, cultivos y traspatio) han sido reportadas para los estados de Aguascalientes y Yucatán, México (MONTIEL et al. 1999; AMADOR y DE LA RIVA, 2016). A pesar de que la cacería en las áreas de producción agrícola obedece a la protección contra los daños ocasionados por las especies plagas, también estos cultivos actúan como trampa de fauna silvestre, por lo que los informantes practican las técnicas de las arriadas o la espiada en estas zonas, principalmente donde se ha sembrado maíz y sorgo, situación similar sucede con la milpa comedero- trampa en comunidades mayas (SANTOS et al., 2013), con la diferencia de que en el área de estudio, la siembra de cultivos no es exclusivamente para atraer y cazar determinadas especies de fauna como sucede en la milpa comedero-trampa maya. 
Respecto a la conservación de la mastofauna local, los habitantes reconocieron que hay especies de mamíferos que han disminuido. Para BERKES (2003), la percepción de disminución de la fauna silvestre por los habitantes locales se deduce de acuerdo al conocimiento adquirido a través de la experiencia en la realización de las actividades cotidianas en el medio natural, tales como la cacería y la práctica agrícola, en el caso particular de los pobladores de Pitzotlan el conocimiento se ha adquirido a través de la realización de diferentes actividades como la recolección de leña y de pitaya, el pastoreo de ganado bovino y caprino, la agricultura de temporal y la cacería. Dicho conocimiento local de los pobladores sobre su entorno, permite obtener una aproximación puntual del aumento o la disminución de especies a través del tiempo (BERKES, 1999).

A pesar de que existen Normas de regulación externas por dependencias gubernamentales y de regulación local acordadas por los habitantes de la comunidad, tales como evitar cazar animales hembras o juveniles, sanciones monetarias u organización de vigilancia comunitaria, estas últimas están dictadas en torno al aprovechamiento del venado cola blanca, olvidando en ocasiones a las demás especies de mamíferos, porque tienen menos importancia económica.

Para el caso del armadillo existe lo que GUERRA et al., (2010) define como la regulación por costumbres, en la que los informantes no consumen el armadillo debido a la creencia de que su carne es venenosa, mito que es reportado por el mismo autor en comunidades de la selva Lacandona, México, situación diferente a lo que sucede en otras partes de México y Sudamérica, donde representa una de las especies más cazadas para consumo (PÉREZ y OJASTI, 1996; DE LA OSSA y DE LA OSSA, 2012). En este tipo de regulación, el conjunto de conocimientos, creencias y costumbres en torno a la fauna está relacionado con la regulación del recurso y se transmite de generación en generación (BERKES, 1999), en el caso de este mito donde los informantes mencionan que data desde 1950. En la comunidad se pueden observar los dos tipos de factores ligados a la regulación de la cacería de subsistencia mencionadas por OSTROM (2001), este autor señala que aquella regulación ligada a los sistemas organizativos como en el primer caso de las reglas acordadas en torno al aprovechamiento del venado cola blanca y la otra regulación está ligada a la cosmovisión de la comunidad por ejemplo en torno al aprovechamiento del armadillo.

La revaloración del conocimiento tradicional de las comunidades campesinas mestizas, es importante (ENRÍQUEZ et al., 2006), e igualmente como lo menciona BARRASA (2012), "el conocimiento tradicional sobre el uso de los recursos naturales se puede tornar como una solución complementaria a parte de los problemas que afectan a las comunidades rurales, especialmente aquellas que se encuentran en condiciones de pobreza y con limitado acceso a servicios públicos básicos"; pero también el registro de los saberes locales constituye un instrumento de análisis para la toma de decisiones pertinentes al diseño de estrategias para el aprovechamiento sostenible y la conservación de un recurso natural, ya que cualquier actividad dirigida al aprovechamiento de la diversidad, debe ser planeada para lograr no sólo el uso, sino también la conservación de los recursos que la constituyen, involucrando la participación y el consenso de las comunidades campesinas e indígenas, los cuales son actores clave para su conservación.

En conclusión el aprovechamiento de mamíferos silvestres en el área de estudio representa una actividad de autoabasto, que permite satisfacer dos necesidades básicas como la alimentación y la salud, además de obtener otros beneficios como estructuras ornamentales o para la fabricación de herramientas.

Para realizar un aporte integral en la toma de decisiones sobre estrategias de manejo, conservación y desarrollo local, es necesario valorar multidisciplinariamente la apropiación de los mamíferos y la fauna silvestre, evaluando aspectos 
García et al - Aprovechamiento tradicional de mamíferos silvestres

socioculturales, ecológicos (tamaño económicas por la venta de presas de

poblacional de las especies presas, biomasa caza) que permitan a partir del rescate, extraída) y económicos (porcentaje de la documentación y análisis del conocimiento economía familiar que se satisfacen por etnozoológico el desarrollo comunitario la apropiación de fauna, costos generados sostenible.

por la cacería, estimación de ganancias

\section{REFERENCIAS}

AMADOR, A.S.; DE LA RIVA, H.G. 2016. Uso tradicional de fauna silvestre en las serranías del occidente del estado Aguascalientes, México. Etnobiología 14 (2):20-36.

ARANDA, S.M. 2000. Huellas y otros rastros de mamíferos grandes y medianos de México. Editorial: Instituto de Ecología México. México.

ARANDA, S.M. 2012. Manual para el rastreo de mamíferos silvestres de México. Editorial: Comisión Nacional para el Conocimiento y Uso de la Biodiversidad. México.

BARRASA, G.S. 2012. Conocimiento y usos tradicionales de la fauna en dos comunidades campesinas de la Reserva de la Biosfera de La Encrucijada, Chiapas. Etnobiología, 10 (1):16-28.

BERKES, F. 1999. Sacred ecology: Traditional ecological knowledge an resource management. Editorial: Taylor and Francis. Estados Unidos de América.

BERKES, F. 2003. Can Cross-Scale Linkages Increase the Resilience of Social-Ecological Systems? Paper presented at the RCSD International Conference, Politics of the Commons, Chiang Mai. Consultado de https://dlc.dlib.indiana.edu/dlc/bitstream/ handle/10535/216/Fikret_Berkes.pdf?sequence $=1$

DE LA OSSA-LACAYO, A.; DE LA OSSA, V.J. 2012. Utilización de fauna silvestre en el área rural de Caimito, Sucre, Colombia. Revista Colombiana de Ciencias Animales 4 (1):46-58.

ENRÍQUEZ, V.P.; MARIACA, M.R.; RETANA, G.O.; NARANJO, P.E. 2006. Uso medicinal de la fauna silvestre en los Altos de Chiapas, México. Interciencia 31 (7):491-499.

ESCAMILLA, A. ; SANVICENTE, L.M.; SOSA, M.; GALINDO, L.C. 2000. Habitat Mosaic, Wildlife Availability, and Hunting in the Tropical Forest of Calakmul, México. Conservation Biology 14 (6):1592- 1601.

GARCÍA, F.A.; LOZANO, G.M.A.; ORTIZ, V.A.L.; MONROY, M.R. 2014. Uso de mamíferos silvestres por habitantes del Parque Nacional El Tepozteco, Morelos, México. Etnobiologia 12 (3):57-67.

GONZÁLEZ, G.F.; LLERENA, R.G. 2014. Cacería de mamíferos en la Zona de Uso Especial y de Amortiguamiento del Parque Nacional Tingo María, Huánuco, Perú. Revista Peruana de Biología 21 (3):283-286.

GUERRA, R.M.; NARANJO, P.E. 2003. Cacería de subsistencia en dos localidades de la selva Lacandona, Chiapas, México. Págs. 317-324. En: Polanco, O. (Ed.), Manejo de fauna silvestre en Amazonía y Latinoamérica. Editorial: CITES-Fundación Natura-Fundación MacArthur. Colombia. 
GUERRA, R.M.; CALME, S.; GALLINA, T.S.; NARANJO, P.E. 2010. Uso y manejo de fauna silvestre en el norte de Mesoamérica. Editorial: Instituto Nacional de Ecología. México.

HAGUETTE, F.T. 1992. Metodologias quantitativas na sociologia. Editorial: Petropolis Voze. Brasil.

HERNÁNDEZ, L.A.; LÓPEZ, A.E.; RODRÍGUEZ, R.A.; AQUINO, B.V. 2013. Diagnostico en el uso de la fauna, en el área de protección de flora y fauna "Cañon del Usumacinta" Tenosique, Tabasco. Ra Ximhai 9 (1):1-13.

INE (INSTITUTO NACIONAL DE ECOLOGÍA . 1997. Programa de Conservación de la Vida Silvestre y Diversificación Productiva en el sector rural. Editorial: SEMARNAP. México.

INEGI INSTITUTO NACIONAL DE ESTADISTICA, GEOGRAFÍA E INFORMATICA. 2010. Censo de población. Instituto Nacional de Estadística y Geografía. Consultado en http:// www3.inegi.org. $\mathrm{mx} /$ sistemas/SCITEL/default?ev $=5$

LIRA, T.I.; BRIONES, S.M.; GÓMEZ-DE ANDA, F.; OJEDA, R.D.; PELÁEZ, A.A. 2014. Uso y aprovechamiento de la fauna silvestre en la Selva Zoque, México. Acta zoológica mexicana 30 (1):74-90.

LÓPEZ, C.C.; ZAZUETA, A.X.; PORRAS, A.C. 2005. Notas sobre el aprovechamiento del medio ambiente nortense: reconocimiento del terreno, inicio de la cacería. Ra Ximhai $1(1): 39-50$.

MARTÍN, C.B.C.; SALAMANCA, C.A. 2007. El muestreo en la investigacion cualitativa. Nure investigacion. Consultado en http://www.nureinvestigacion.es/OJS/index.php/nure/ article/view/340

MIRANDA, G.F.; HERNÁNDEZ, X.E. 1963. Los tipos de vegetación de México y su clasificación. Boletín de la Sociedad Botánica de México 28 (1):29-179.

MONROY, M.R.; GARCÍA, F.A. 2013. La fauna silvestre con valor de uso en los huertos frutícolas tradicionales de la comunidad indígena de Xoxocotla, Morelos, Mexico. Etnobiología 11 (1):44-52.

MONROY, V.O.; CABRERA, G.L.; SUÁREZ, H.P.; ZARCO, G.M.; RODRÍGUEZ, S.C.; URIOS, M.V. 2008. Uso tradicional de vertebrados silvestres en la Sierra Nanchititla, México. Interciencia $33(4): 308-313$.

MONTIEL, O.S.; ARIAS, R.L.; DICKINSON, B.F. 1999. La cacería tradicional en el norte de Yucatán: una práctica comunitaria. Revista de Geografía Agrícola 29 (1):29-42.

MUNDO, S.F. 2011. Municipios y localidades marginadas de Morelos: Notas sobre la historia reciente. Editorial: Consejo Estatal de Población. México.

NARANJO, E.; GUERRA, M.; BOLAÑOS, R. 2004. Subsitence huting by three ethnic group of the lacandon forest, México. Journal Ethnobiology 24:233-253.

OJASTI, J.; DALLMEIER, F. 2000. Manejo de Fauna Silvestre Neotropical. Editorial: Smithsonian Institution/MAB Biodiversity Program. Estados Unidos de América. 
ORGANIZACIÓN PANAMERICANA DE LA SALUD. 2003. Clasificación Estadística Internacional de Enfermedades y Problemas Relacionados con la Salud. Consultado en http://iris. paho.org/xm/ui/bitstream/handle/123456789/6282/Volume1.pdf?sequence =1

OSTROM, E. 2001. El gobierno de los bienes comunes. La evolución de las instituciones de acción colectiva. Editorial: Universidad Nacional Autónoma de México/Fondo de Cultura Económica. México.

PARRA, C.J.W.; BOTERO, B.A.; SAAVEDRA, R.C.A. 2014. Percepción y uso de mamíferos silvestres por comunidades campesinas andinas de Génova, Quindío, Colombia. Bol. Cient. Mus. Hist. Nat. U. de Caldas.18 (1):78-93.

PAUTASSO, A. 2003. Aprovechamiento de la fauna silvestre por pobladores rurales de la fracción norte de los bajos submeridionales de la provincia de Santa Fe, Argentina. Editorial: Museo Provincial de Ciencias Naturales "Florentino Ameghino". Argentina.

PÉREZ, E.; OJASTI, J. 1996. La utilización de la fauna silvestre en la América Tropical y recomendaciones para su manejo sustentable en las sabanas. Ecotropicos 9:71-82.

RACERO, C.J.; GONZÁLEZ, M.J. 2014. Inventario preliminar y uso de mamíferos silvestres por comunidades campesinas del sector oriental del cerro Murrucucú, municipio de Tierralta, Córdoba, Colombia. Mammalogy Notes 1 (2):25-28.

RETANA, G.O.G.; AGUILAR, N. M.; NIÑO, G.G. 2011. Uso de la vida silvestre y alternativas de manejo integral: el caso de la comunidad maya de Pich, en Campeche, México. Tropical and subtropical Agroecosystems 14 (3):885-890.

RETANA, G.O.G.; MARTÍNEZ, P.L.; NIÑO, G.G.; VICTORIA, C.E.; CRUZ, M.A.; UC-PIÑA, A. 2015. Patrones y tendencias de uso del venado cola blanca (Odocoileus virginianus) en comunidades mayas, Campeche, México. Therya 6 (3):597-608.

ROBLEDO, M.J. 2009. Observación participante: informantes claves y el rol del investigador. Nure Investigación 42(6):1-4.

RODAS, T.J.; OCAMPO, G.P.; COUTIÑO, H.P. 2014. Uso de los mamíferos silvestres en el municipio de Copainalá, región Zoque, Chiapas, México. Quehacer Científico en Chiapas 9 (2):3-9.

SANTOS, F.D.; NARANJO, P.E.; BELLO, B.E.; ESTRADA, L.I.; MARIACA, M.R.; MACARIO, M.P. 2013. La milpa comedero-trampa como una estrategia de caceria tradicional maya. Estudios de cultura maya 42 (1):89-118.

STEARMAN, A.; REDFORD, K. 1995. Game management and cultural survival: The Yuquiethno development project in lowland Bolivia. Oryx 29 (1):29-34.

TAYLOR, S.J.; BOGDAN, R. 1986. Introducción a los métodos cualitativos de investigación. Editorial: Paidós. España.

TOLEDO, M.V.; BARRERA, B.N.; GARCÍA, F.E.; ALARCÓN, C.P. 2008. Uso Múltiple y Biodiversidad entre los Mayas Yucatecos. México. Interciencia 33 (5):345-362.

VELARDE, E.S.; CRUZ, L.A. 2015. La fauna silvestre y su relación con el bienestar de tres comunidades de la Reserva de la Biosifera Sierra de Huautla, Morelos. Etnobiología $13(1): 39-52$. 